\title{
Berliner augenärztliche Gesellschaft
}

\section{Sitzung vom 25. April 1929}

A. Gutmann: Demonstration eines weiteren Falles von charakte-ristischer Sehnervencxkavation bei Hypopliysistumor, dieses Mai an einem nicht myopischen Auge. G. halt die von ihm beobachtete trichterför-mige Exkavation in der nasalen Sehnervenhälfte für charakteristisch bei Hypophisistumoren, entstanden durch Schrumpfung in der Längs-richtung der Sehnerven.

Diskussion :

Comberg konnte in dem Falle von G. keine ungewöhnliche Ver-änderung mit dem Augenspiegel erkennen.

Ginsberg halt die in der vorigen Sitzung gezeigte Papille nicht für atrophisch, sondern für myopisch bei schräger Stellung des Sehnerven-kopfes. Eine Trichterbildung in der Papille kann nicht auf Schrumpfung von Fasern im Sehnerven zurückgeführt werden. Die Abblas-sung der Sehnerven ist durch Wucherung der Glia zu erklären.

Gutmann: Da die Lochbildung im Sehnerven tiefer ist' als die myopische Exkavation. bleibt zur Erklärung nur die Annahme eines Zuges von hinten her übrig.

Levinsohn: Zur Kritik der Myopiegenese. Gegenüber den Anschau-ungen Scheerers und Bucklers halt L. an seinen Thesen fest. Der Beweis für seine Anschauungen sind für ihn :

i. Die anatomischen Veränderungen bei Myopie,

2. die Tatsache, daß diese $\Lambda 1 / 8$ ränderungen nicht angeboren sondern fast ausnahmslos erworben sind. Sie fehlen beim jungen Kinde und entwickeln sich erst, wenn das Wachstum des Auges fast abgeschlossen ist. Sie entstehen durch allmählkhe Dehnung, den senilen Veränderungen können sie ähneln, sind aber anderer Art. Sie enstehen bei stärkerer Kopf-und Rumpfbeugung, zum geringen Teil wird der Erbfaktor anerkannt.

Diskussion :

Bucklers zeigt seine anatomischen Präparate, aus denen er nachweist, daß dieselben Veränderungen, die als Beweis für die Myopieentstehung angeführt werden, sich auch in nicht myopischen Augen, hyperopischen und senilen Augen vorfanden.

Levinsohn halt die Anschauungen von Bucklers für widerlegt. Die Befunde bei der experimentellen Affenmyopie seien allein bewei-send für die Entstehung der Myopie. Kleiber: $\Lambda$ kustiseh-chromatisehe Synopsien. Kl. bringt eigene Beob-achtungen zur Erscheinungsweise der Synopsien. Bei ihm erreichen Vokale und Zahlen Farbeneindrücke, und zwar :

$\mathrm{A}=$ schwarz, $\mathrm{E}=$ weiß, $\mathrm{I}=$ gelb, $\mathrm{O}=$ braun, $\mathrm{U}=$ blau, $\mathrm{Al}=$ grau, $\mathrm{Oe}=$ hellbraun, $\mathrm{Ue}=$ hellblau, $\mathrm{Au}-$ rot, $\mathrm{Eu}=$ grün, $\mathrm{Au}=$ graugrün,

$\mathrm{Ei}=$ strahlend weiß.

Zahlen : $\mathrm{i}=$ dunkelgrau, 2 - hellgrau, $3=$ weiß, $4=$ blau, 5 grün, $6=$ blaugelb, $7=$ sattgelb, $8=$ rot, $9=$ schiefergrau, $10=$ schwarz, $11=$ hellbraun, 12 = dunkelbraun.

Berliner augenärztliche Gesellschaft. 
Der Farbeneindruck tritt auf beim Klang wie bei der bloßen Vorstellung der Zahl bezw. Vokal. Die Projektion der Farbe er-folgten nach außen. Die Empfindungen sind seit frühester Kindheit vorhanden und ko $\pi$ stant. Bei anderen Personen werden ähnliche Erscheinungen durch Tone hervorgerufen.

Es handelt sich bei diesen Erscheinungen nicht urn krankhafte, sondern physiologische Vorgänge. $12 \%$ aller Menschen haben sie. Von alien Erklärungen ist diejenige Langenbecks am warscheinlichsten, daß es sich hierbei um Assoziationen aus frühester Kindheit handle. Unerklärlich bleibt die Tatsache, daß dieselben Reize bei verschie-denen Synoptikern verschiedene Farbenempfindungen auslösen.

Diskussion ;

Paderstein fragt, ob Untersuchungen über die Beziehungen zwischen Synopsie und Eidetik vorliegen. Goethe war vermutlich Synoptiker und Eidetiker.

Comberg regt an, Farbenmischungen auszuführen aus der synop-tische $7 / 8$ und einer objektiv gewählten Farbe. Es ware denkbar, daß aus dem Vergleich mit den Verhältnissen b i der monokularen und der binokularen Farbenmischung Schlüsse zu ziehen wären hinsichtlich der Lokalisation und der Deutung der Vorgänge.

Czellitzer glaubt nicht, daß bei den Synopsien Gesetzmäßigkeiten gefunden werden können, wem die Vorgänge auf Assoziationen aus früher Jugend beruhen. Dem Nicht-Synoptiker werden die Vorgänge immer als etwas Willkürliches und Phantastisches erscheinen.

Kleiber (Schlußwort): Goethe war zweifellos Synoptiker. Ein Zu-sammenhang z $\lambda$ vischen Synopsie und Eidetik ist unbekanrt. Kl. selbstist nicht Eidetiker. Combergs Ariregurg ist dankenswert, er selbst seiaber kein geeignetes Versuchsobjekt. Dr. A. Rosenberg. Verein der Augenärzte von Ost- und $\Lambda$ Vestpreußen.

Sitzung vom 24. November 1928 in der Universitäts-Augenklinik Königsberg i. Pr. Frl. Gredsted, Königsberg i. Pr.: Die tonometrisehe Analyse beim Glaukom. (Erscheint ausführlich in dieser Zeitschrift.) K.v. von Jarmersted referiert an Hand eines Falles über Sehstòrung • (lurch Blutverlust. Bei einem 49jährigen

vorher stets gesund gewesenen Patienten stellte sich im Anschluß an eine starke Darmblutung ver-bunden mit Hämatemesis infolge eines Duodenalulkus nach etwà 13 Stun-den eine fast vollständige Amaurose beider Augen ein. Bei der Ein -lieferung des Patienten in die Augenklinik (drei Monate nach dem Blutverlust) zeigt der Augenhintergrund eine deutliche Blässe der temporalen Papillenhälften außerordentliche Enge der Gefäße sowie perimakuläre Lichtungen und feine Marmorierung. Geringe zentrale Pigmentver- 